\title{
Quaestiones disputatae
}

\author{
Jean-Pierre Angoujard \\ Laboratoire de Linguistique de Nantes (LLING) \\ jean-pierre.angoujard@univ-nantes.fr
}

\section{Tout est transparent}

Nous admettrons que la notion de contrainte est retenue par tous. Pour avoir lu le dernier ouvrage de J. J. McCarthy (McCarthy, 2007), nous n'admettrons pas que le recours aux dérivations ait été abandonné de tous.

La plus noble tâche du phonologue consisterait-elle à rendre compte des « généralisations cachées » (Hidden generalizations) ? Il me semble que l'usage du terme de «généralisation » mérite d'être assez largement commenté : de son interprétation ressortiront nos conceptions de la phonologie. Par contre, je ne m'attarderai pas exagérément sur la quête de la généralisation cachée pour une simple raison : est caché ce que l'on décide de représenter comme caché (par exemple parce qu'on a du goût pour les dérivations et les représentations sous-jacentes). Il se trouve également que les exemples cruciaux retenus et analysés par McCarthy sont des données puisées dans la dialectologie de l'arabe et ne concernent donc qu'indirectement le propos de ce congrès.

Quel est le débat? Dans l'arabe de Damas (comme en latin) et pour prendre un seul exemple, l'accent de mot porte sur la pénultième si elle est lourde ( $c f$. [katábna] «nous avons écrit ») et sur l'antépénultième si la pénultième est légère $(c f$. [kátabu] «ils ont écrit »). L'accentuation des syllabes en position finale de mot et dites « surlourdes » $(c f$. [katábt] «j'ai écrit») peut être rapportée à l'accentuation des pénultièmes lourdes : [katábt*]. Il se trouve que cette forme de 1ère personne du singulier est également réalisée comme [katábət]. On notera que, dans cette dernière forme, la voyelle [ə] est clairement une réalisation par défaut, distincte des schwas accentuables qui font partie intégrante du système vocalique de l'arabe de Damas (cf. [Yámel] « il a fait»), et qui n'apparaissent jamais dans la dernière syllabe du mot.

Il n'est aucunement inévitable de recourir à un ordre accentuation $\rightarrow$ épenthèse. Il est tout d'abord possible de se référer à un phénomène d'interprétation phonétique (au sens de Coleman, 1998), plus précisément dans ce cas à une interprétation temporelle (figure ci-dessous) et la question, alors, n'est plus phonologique.

\begin{tabular}{|c|c|c|c|c|}
\hline$<a>$ & \multicolumn{2}{|c|}{$<\mathrm{a}>$} & \multicolumn{2}{|l|}{$\mathrm{v}_{\text {def }}$} \\
\hline$<\mathrm{k}>$ & $<\mathrm{t}>$ & $<b>$ & $<t>$ & [katábt’ \\
\hline$<\mathrm{a}>$ & & & $\mathrm{v}_{\text {def }}$ & \\
\hline$<\mathrm{k}>$ & $<\mathrm{t}>$ & $<\mathrm{b}>$ & $<t>$ & $=\left[\mathrm{katáb}{ }^{\urcorner}\right]$ \\
\hline
\end{tabular}

Il est également possible (et je préfère de loin cette approche) de se référer à une hiérarchie vocalique incluant les deux types de sommets réalisés schwas à Damas (représentés ci-dessous comme v, pour les schwas accentuables, et $\mathrm{v}_{\text {def }}$, réalisation par défaut d'un sommet non lexicalement instancié) :

$$
\begin{aligned}
& \mathrm{V} \\
& 3
\end{aligned}>\mathrm{v}>\begin{gathered}
\mathrm{v} \\
2
\end{gathered} \quad \begin{gathered}
\text { def } \\
1
\end{gathered}
$$

La notion de syllabe lourde a été rapportée dans Angoujard (2006) à un objet $m o d \_H$ (pour « modèle rythmique lourd ») qui inclut non seulement les séquences [CVC] ou [CV:], mais également les séquences 
[CVC*]. On retiendra alors pour Damas une version élargie de l'objet $m o d \_H$ en y intégrant la description (contrainte) suivante :

$$
\text { C_mod_H }:=\left\{\bmod _{-} L \wedge\left(\bmod _{-} L|\operatorname{som}| \operatorname{Seg}_{[1]} \wedge([<2))\right\}\right.
$$

Cette contrainte n'a rien de mystérieux, bien au contraire. Elle revient tout simplement à reconnaître que le locuteur damascène interprète comme rythmiquement identiques les séquences $\left[\mathrm{CVC}^{*}\right]$ et $\left[\mathrm{CVCv}_{\text {def }}\right]$, ce qui est bien le cas puisqu'il accentue [katáb*t] comme [katábət]... Et on ne voit pas pourquoi la phonologie devrait être compliquée.

\section{Des généralisations substantielles}

Il n'est pas rare d'entendre confronter une phonologie « descriptive » (celle qui se définit elle-même plutôt comme «déclarative »- le structuralisme aussi a essuyé ce reproche) à des phonologies qui sauraient lire au-delà des apparences (optimales, du gouvernement). Il n'est pas rare non plus d'entendre confronter des phonologies « abstraites », « symboliques » (déclaratives, optimales, du gouvernement) à des phonologies plus substantielles (que cette substance soit celle de l'articulatoire ou du neuronal).

Il me semble que la phonologie doit, modestement, approcher les généralisations transparentes de la langue et que, d'autre part, elle ne peut échapper à la substance. Il ne reste plus qu'à s'interroger : quelles généralisations? quelles substances? (peut-être aussi serait-il pertinent d'ajouter : quelle langue ? mais cette dernière question émergera certainement quand sera développé, ici même et par plus compétent que moi, l'objet « corpus »).

La mise en évidence de généralisations commence bien évidemment par des observations. Ainsi, s'est-on avisé, pour le moins depuis la fin du XIX ${ }^{e}$ siècle, qu'en français il existait des contraintes sur les successions de consonnes. Une généralisation, donc : la «loi des trois consonnes » (Grammont, 1894). Il a été

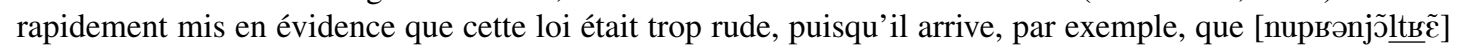
(«nous prenions l'train »)... La contrainte, on le sait, se doit donc également de prendre en compte la qualité rythmique (syllabique) des consonnes (dans notre exemple, [tь] constitue une attaque double), ce qui revient à se soucier de la nature des consonnes puisque n'est pas en deuxième position d'une attaque double qui veut. Autrement dit, la généralisation a dû affiner notablement sa prise en compte de la substance.

Assez curieusement, si la version de la théorie du gouvernement retenue dans Charette (1991) prend bien en compte, à sa manière très particulière, la substance (dans le mot [guvعsnəmã] 《gouvernement », la consonne $[\mathrm{b}]$, en position de coda, doit être gouvernée par le [n]), la structure syllabique n'y est pas considérée comme cruciale (mais cette force obscure qu'est le gouvernement) et l'auteur rejette bien à tort le [guvesnmã] «gouvern'ment » (voir, pour une analyse critique complète, Lyche \& Durand, 1996).

L'apparition (si on peut dire...) des « positions vides» (je reviendrai plus loin sur cet objet), et, plus spécifiquement, l'analyse rythmique proposée dans Angoujard (1997) qui accorde toutes leurs places aux attaques doubles et aux sonantes en coda, a permis de remplacer la contrainte sur la succession des trois consonnes par une contrainte sur la succession immédiate de deux sommets vides : dans tous les cas un sommet, au moins, sera instancié et, si nécessaire, il le sera, par défaut, comme schwa (pour la discussion de quelques données et contre-exemples éventuels en français et en cadien, voir Angoujard, 2006, p. 90-91). Cette contrainte peut naturellement être représentée et intégrée à la grammaire du français, par exemple, sous la forme d'une structure de traits typés : 


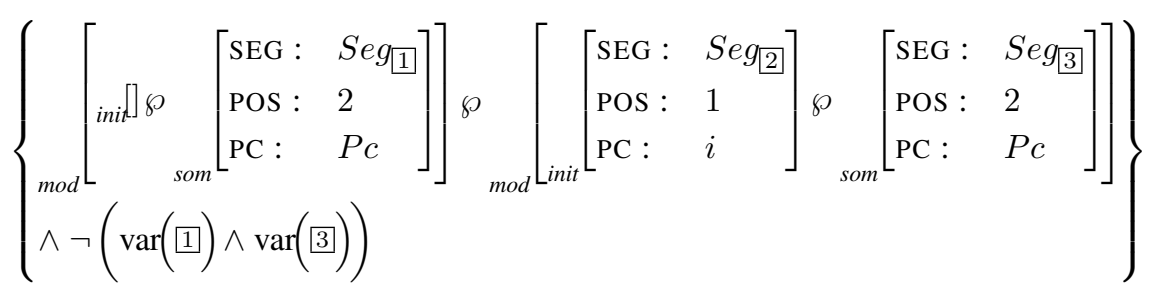

Une question demeure : pour quelle raison cette contrainte est-elle présente dans la grammaire du français ?

La réponse, pensons-nous, est prosodique, plus précisément, rythmique. Selkirk a présenté, il y a tout juste 30 ans, un modèle de la structuration rythmique du français qui reste, à de nombreux égards, le plus pertinent : elle suppose que le français construit, pour l'essentiel, des pieds unaires (constitués d'une seul syllabe) et que les seuls pieds binaires qui s'y rencontrent sont de type [+-] (trochaïque) incluant en position faible (donc à droite) une syllabe dont le sommet est associé à un schwa (Selkirk, 1978). Le schwa étant aujourd'hui interprété comme la réalisation (contrainte) d'un sommet dit « vide », on étendra naturellement la description des pieds binaires du français à ceux dont le sommet en position faible est non lexicalement instancié ou, si l'on préfère, aux sommets dont le trait SEG a pour valeur la disjonction (ə $\vee S e g$ ).

Ainsi, dans une séquence $\left[\sigma \sigma \sigma_{v} \sigma \sigma\right]$ (où $\sigma_{v}$ représente une syllabe ou modèle rythmique dont le noyau est lexicalement « vide »), on s'attendra à rencontrer la structuration en pieds suivante (on rappellera qu'une structure contenant 6 pieds unaires est également possible, sous réserve d'autres contraintes lexicales et/ou prosodiques imposant l'une ou l'autre des structures) :

$$
\left[\begin{array}{lllll}
\sigma & {[\sigma} & \sigma_{v} & {[\sigma]} & {[\sigma]}
\end{array}\right]
$$

Si la séquence inclut deux syllabes ou modèles rythmiques consécutifs à noyau lexicalement vide, seules les deux structurations représentées ci-dessous sont envisageables (on se rappellera que si, en français, la position faible d'un pied binaire est toujours occupée par un sommet lexicalement non instancié, rien n'interdit à un tel sommet d'être en position forte dans un pied binaire avec, bien sûr, un autre sommet lexicalement non instancié en position faible). Or, si on admet qu'un noyau en position rythmique forte (dans un pied binaire ou unaire) se doit d'être instancié, il n'existe alors aucune solution, en français, pour que deux noyaux vides adjacents demeurent non instanciés pour SEG et la contrainte imaginée pour les sommets vides se révèle absolument superflue.

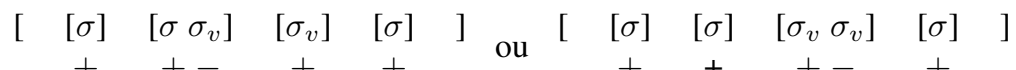

Les seules généralisations pertinentes, ici, font donc partie de celles, fort «générales », qui gouvernent l'organisation rythmique du français et la nature des sommets syllabiques. Pour ces sommets, on retiendra par exemple une description du type som (pour sommet) intégrant la contrainte sur la nécessaire instanciation de la valeur du trait SEG lorsque le poids prosodique de ce sommet (partiellement calculé à partir de l'organisation en pieds et représenté par un trait [POIDS : Poids]) est supérieur à 2 (i.e. instancié) :

$$
\left\{\left[\begin{array}{ll}
\text { SEG : } & \text { Seg } \\
\text { POS : } & 2 \\
\text { PC : } & \text { Pc }
\end{array}\right] \wedge((\text { Poids }>2) \Rightarrow(\neg \operatorname{var}(\text { Seg })))\right\}
$$




\section{La substance du vide}

Il est d'usage, en un premier cours pour jeunes phonologues francophones, de solliciter les découpages syllabiques respectifs des deux mots [pasti] «parti » et [patsi] «patrie ». La réponse étant immédiate et attendue, ceci permet à l'enseignant de faire remarquer aux étudiants combien ils sont (déjà !) d'excellents linguistes et de passer ensuite au «pourquoi ?» de ces découpages... avec pour toute réponse, souvent, un silence éloquent.

Les étudiants seront ensuite guidés vers les notions de coda, d'attaque et d'attaque double et vers la distinction entre les occlusives et les liquides. On peut tourner cela dans tous les sens, mais ces deux catégories de consonnes sont substantiellement distinctes. Et c'est bien cette opposition de substance qui gouverne la répartition en [coda-attaque] vs [attaque double].

Dans une vie presque antérieure (c’était au début des années 80), je présentais à une assistance très «phonéticienne » un découpage bisyllabique de la forme verbale [ktıb] «il a écrit» en arabe de Tunis (soit [k-tıb]. Certes, le terme (bi)syllabique était mal choisi (j'y ai renoncé plus tard) dans la mesure où pour compter les syllabes tout un chacun, au moins en français, compte les voyelles réalisées et qu'il n'était pas dans mon projet d'affirmer que [ktıb] comprend deux voyelles... Je faisais bien plutôt référence à la présence d'un noyau vide entre le [k] et le [t], mais les phonéticiens ont fort justement horreur du vide.

$\mathrm{J}$ 'aurais mieux fait de dire que les deux occlusives $[\mathrm{k}]$ et $[\mathrm{t}]$ sont des attaques (cette dernière aussi bien dans [ktıb] que dans [pasti] et [patsi]). Il est ensuite aisé, me semble-t-il, d'argumenter sur le fait que la séquence $[\mathrm{kt}]$ n'est pas comparable à une séquence $[\mathrm{tr}]$ (ou alors il faut négliger les connaissances de nos étudiants), ni à une séquence $[\mathrm{st}]$ ou [rt] : la réalisation d'une forme verbale comme [rtah] «il s'est reposé », toujours en arabe de Tunis, implique que la première consonne (liquide) regarde à gauche, avec des réalisations de type [ ${ }^{\ominus}$ rtah] ou [rtah] (ce qui est exclu en français, mais que le gallo, comme l'arabe maghrébin, permet une parenthèse substantielle pourrait faire remarquer qu'arabe et gallo font usage d'un $[\mathrm{r}]$ apical quand le français retient aujourd'hui majoritairement un $[\mathrm{b}]$ uvulaire).

On se rappellera qu'une occlusive, à tout le moins en français et en arabe, est systématiquement réalisée ouvrante. Et dire qu'il existe un noyau (sommet) vide entre le [k] et le [t] dans la séquence [kttb] peut donc se traduire ici très simplement par « $[\mathrm{k}]$ est une occlusive (ouvrante) ». Ce qui, j'imagine, n' aurait guère suscité de controverse...

Soit :

- les représentations du type [k*tib*] (où la position vide est rendue visible);

- les représentations plurilinéaires :

$\begin{array}{cccccc} & \mathrm{x} & & \mathrm{x} & & \mathrm{x} \\ \mathrm{x} & \mathrm{x} & \mathrm{x} & \mathrm{x} & \mathrm{x} & \mathrm{x} \\ \mid & & \mid & \mid & \mid & \\ \mathrm{k} & & \mathrm{t} & \mathrm{I} & \mathrm{b} & \end{array}$

- les représentations en structures de traits (ici simplifiée, mais on appréciera le fait que cette représentation associe à la position vide une variable, autant dire tout un choix substantiel) :

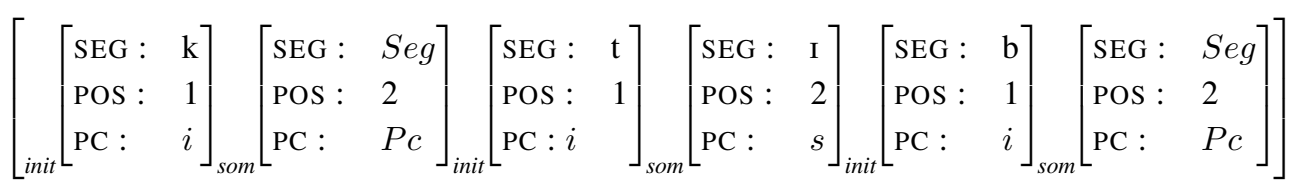

Que faut-il penser de ces représentations ? Il faut en penser qu'elle sont strictement identique à [ $\left.\mathrm{k}^{\ulcorner} \mathrm{t}^{\ulcorner} \mathrm{I} \mathrm{b}^{\ulcorner}\right]$.

Remarque : Je ne pense pas, comme cela a pu être argumenté (Brandão, 2004), qu'il faille opposer les $\overline{\text { séquences }}[\mathrm{kt}]$ en latin (où $[\mathrm{k}]$ serait coda) et en arabe (où les deux occlusives seraient séparées par une position vide); le [k] latin (de [kt]) s'est substantiellement modifié (articulatoirement et/ou en perception) 
jusqu'à produire les réalisations [tt] de l'italien et [jt] du français qui, à l'évidence, et pour l'interprétation que j'en fais, n'autorisent pas de représentations avec positions vides. J'admettrai (et certains mesureront l'effort, si ce n'est le progrès) qu'à une certaine période du latin, le son représenté comme [k] a pu être en position de coda (i.e. sans noyau vide à sa droite). Mais il s'agissait alors d'un objet distinct du [k] ouvrant de $\left[\right.$ fak $\left.^{r} t^{\top} u\right]$, disons un très provisoire $\left[k^{\urcorner}\right]$vite « sonorisé ».

\section{Prendre la parole}

Il est tout à fait regrettable que les chercheurs en «parole» (ceux qui se retrouvent tous les deux ans aux JEP) et les chercheurs phonologues aient, souvent, bien peu en commun (combien de phonologues patentés ont déjà participé aux JEP ?). Même les « Journées » régulièrement organisées à Nantes, si elles ont permis des écoutes respectueuses et des échanges, n'ont que bien rarement débouché sur des travaux communs.

Je dois avouer que j'ai mis longtemps à me rendre compte que les fondateurs de la Phonologie Déclarative entendaient par «phonologie »... une phonologie ! Je veux dire, quelque chose de distinct d'une «phonétique ». Et que cette phonétique, si on devait s'y référer, se trouvait mystérieusement en dehors du niveau unique de représentation revendiqué par l'approche déclarative. La conséquence est d'importance puisqu'on se retrouve, de fait, tenu de tracer une frontière entre ce qui relève de l'analyse phonologique et ce qui appartient, peut-être, au signal. J'ai déjà fait allusion à la position et pratique de Coleman qui établissait un véritable mur entre les représentations phonologiques et l'interprétation phonétique : "The non-derivational approach to phonology demands a rigorously maintened distinction between phonology, on one hand, and phonetics, on the other." (Coleman, 1998, p. 169), aussi : "Phonological representations are purely formal, constructed in an algebraic model, whereas phonetics representations are descriptions of continuous events, constructed in teh physical domain.” (idem, p. 176).

La question qui surgit immédiatement est de savoir comment distinguer ce qui revient au phonologique de ce qui revient au phonétique. L'opposition continu vs discontinu ne suffit pas : la courbe prosodique (de sonorité) est un objet continu ; la grille rythmique elle-même peut-être interprétée comme la manifestation d'une fonction (au moins s'agit-il d'un débat qui sera notamment ouvert l'an prochain, à l'occasion des

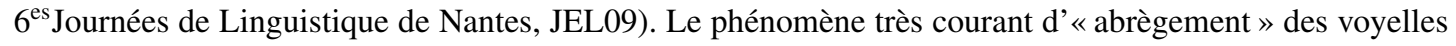
longues non accentuées, est-il phonétique? L'alternance [katábt] vs [katábət], est-elle phonétique?

J'ai eu l'occasion de dire (sans oser l'écrire jusqu'ici) que «le signal n'existait pas » (dans un exposé à l'école d'été de 2003, repris dans Angoujard, 2005). Cela met simplement en avant, avec brusquerie, le fait que tout signal de parole, dès qu'il est produit ou entendu est interprété. Mais, en phonologie déclarative, cette conception a une conséquence : ce signal interprété n'est pas distinct de sa représentation globale sous formes de contraintes. On peut naturellement penser que bien des informations qui doivent être communiquées à un système de synthèse de la parole n'ont pas à être pris en compte par la phonologie/phonétique. C'est vrai dans la mesure où ces informations sont nécessité par la machine et ses propres contraintes (qui ne sont pas celles du cerveau/appareil vocal humain). On peut aussi se demander si les détails qui permettent d'identifier une voix particulière (au sein d'un même groupe linguistique) doivent être pris en compte. Il y a probablement plus urgent, plus essentiel, plus général. Mais en droit (et même en substance) ?

Il est assuré que si je représente comme [SEG : $\{\mathrm{A}, \mathrm{I}\}]$ la voyelle représentée en API comme $[\varepsilon]$, je suis loin d'avoir fourni toutes les informations (en particulier celles qui dépendent du contexte consonantique, ou du contexte prosodique). Mais il n'est aucunement exclu de proposer une notation pour certaines de ces informations (en terme d'unification), au moins pour les plus évidentes. Et nous ne devons pas oublier non plus que le symbole $[\varepsilon]$ ne représente pas un point dans l'espace vocalique, mais un ensemble (un nuage). Toute représentation peut être (sera) incomplète, cela ne récuse pas son adéquation (identité) de principe à la substance phonique. 


\section{Prosodie. Quoi d'autre?}

Le rythme est prosodie. Reconnaître une spécificité des consonnes initiale et finale de [ktıb], reconnaître une organisation rythmique [k-tI-b], revient à prendre en compte une représentation en domaines, soit, minimalement (avec $\sigma$ pour «syllabe » ou modèle rythmique, $\Sigma$ pour «pied» et $\omega$ pour «mot prosodique ») :

$$
\omega\left(\Sigma\left(\sigma(\mathrm{k}){ }_{\sigma}(\mathrm{ti})_{\sigma}(\mathrm{b})\right)\right)
$$

Au-delà, nous admettrons, dans la ligne des propositions de la phonologie prosodique (Nespor \& Vogel, 1986) que toute séquence réalisée est analysable en un ou plusieurs syntagmes phonologiques (de type phrase, noté $\Phi$ ). Ainsi, par exemple, l'accentuation en français aura pour domaine le syntagme phonologique. De nombreux processus phonologiques (tous ?) ont pour lieu d'action un domaine prosodique dont l'extension est variable. Le processus de liaison, en français, est exemplaire. Dans le cadre retenu dans Angoujard (2006), la liaison suppose un partage de valeur entre les traits DOM des mots liés, ce partage n'étant permis qu'à l'intérieur d'un domaine de liaison (ci-dessous représenté comme $\delta$ ) :

$$
{ }_{\delta}(\ldots \text { mot } \mid \mathrm{DOM} 10 \text { mot } \mid \mathrm{DOM}: \text { liaison } 1 \ldots)
$$

Une des communications présentées à ce congrès et intitulée «La liaison 'obligatoire' avec et sans enchâ̂nement $\gg$ est particulièrement pertinente pour notre propos. On se rappelle que les liaisons enchaînées en contexte de rupture intonative y sont analysées comme le résultat de deux contraintes compatibles et coexistantes se référant à deux domaines : celui du « groupe intonatif » $(\Phi)$ et celui du « contexte de liaison » $(\delta)$. On pourrait alors représenter ainsi la séquence $\{J$ 'en ai un [pəti- t] éléphant $\}$, sous la forme d'un chevauchement de domaines $(\Omega$ vaut pour «énoncé ») :

$$
\Omega\left({ }_{\Phi}\left({ }_{\delta}\left(J^{\prime} e n a i\right){ }_{\delta} \delta(\text { un }[\text { pəti- } \mathrm{t}])_{\Phi} \Phi(\text { éléphant })_{\delta}\right)_{\Phi}\right)_{\Omega}
$$

Séquence qui se distinguerait d'une réalisation sans rupture intonative :

$$
\Omega\left({ }_{\Phi}\left({ }_{\delta}\left(J^{\prime} e n ~ a i\right){ }_{\delta} \delta(\text { un petit éléphant })_{\delta}\right)_{\Phi}\right)_{\Omega}
$$

J'ai proposé ailleurs (Angoujard 2006) que cet usage du domaine (et du trait [DOM : Dom]) soit généralisé, avec, pour valeur, une chaîne de caractère. Si ce trait a évidemment pour valeur liaison lorsqu'il fait partie de la description d'un mot autorisant, en position de мот2, la liaison en français (ami, mais ni garçon, ni onze), il peut avoir une valeur harm_voc (pour «harmonie vocalique»), comme dans les exemples du français étudiés dans Nguyen \& Fagyal (2003). La représentation ci-dessous correspond à l'adjectif « dévot» « dévote». La présence du trait DOM avec pour valeur harm_voc conduira à l'application d'une contrainte d'harmonie au sein des bisyllabes (contraignant ici la valeur des traits TETE) avec, pour résultat ([devo] [devot]).

$$
\left[\begin{array}{l}
\langle\mathrm{d}\rangle \wp\left[\begin{array}{ll}
\text { PLACE }: & \langle\mathrm{I}, \mathrm{A}\rangle \\
\text { TETE }: & \text { Tete }
\end{array}\right] \wp\langle\mathrm{v}\rangle \wp\left[\begin{array}{ll}
\text { PLACE }: & \langle\mathrm{U}, \mathrm{A}\rangle \\
\text { TETE }: & \text { Tete }
\end{array}\right] \wp\langle\mathrm{t} \vee \text { a }\rangle \\
\wedge \text { DOM }: \text { harm_voc }
\end{array}\right]
$$

Un même usage du trait DOM (et, bien sûr, de la structuration en domaines prosodiques) peut être retenu pour l'analyse des mutations consonantiques du breton, qui définira plusieurs valeurs pour ce trait (spir, plus, moins) correspondant aux diverses formes de mutations reconnues (spirante, adoucissante, durcissante dans la terminologie traditionnelle). Exactement comme pour la liaison, ces processus (en fait la résolution de disjonctions et l'instanciation de variables) ont lieu au sein d'un domaine prosodique, ici le plus souvent de type : ${ }_{\delta}$ ( clitique + nom ou verbe). 
D'une manière quelque peu simplificatrice, on pourrait opposer une théorie syntaxique de la phonologie (la théorie du gouvernement) à une théorie prosodique de la phonologie (sans doute cette dernière expression est-elle redondante). Une illustration de cette opposition peut être trouvée dans la comparaison entre les analyses de l'article défini en hébreu et en français dans Lowenstamm (1999) et dans Angoujard (2006), dont je rappellerai ici deux phrases extraites de la conclusion du chapitre concerné : "Son statut de clitique impose à l'article défini une structure rythmique minimale, i.e. celle d'un unique modèle rythmique. Le choix [...] d'un modèle rythmique à 2 ou 3 positions est déterminé par la nature du segment lexical associé. ».

Quelle que soit la préférence du phonologue (et indépendamment de la validité des arguments retenus), on reconnaîtra que la position défendue dans Angoujard (2006) est prosodique ( $c f$. le mot « clitique ») et construite sur la substance ( $c f$. « rythmique », « nature du segment »).

\section{Un mot, peut-être}

Un mot, peut-être, sur (hors de ?) la Grammaire Universelle. L'interprétation de l'objet «syllabe » comme le résultat de l'interaction entre la grille rythmique, la courbe prosodique et les segments a été conçue avec la conviction que l'organisation prosodique du langage (sa phonologie ?) était construite sur une adaptation entre le rythme imposé par notre perception (par notre système cognitif) et les caractéristiques physiques des sons de la parole (Angoujard, 1997). Il se pourrait, plus généralement, que les caractéristiques même des représentations construites soient, tout simplement (?), celles permises par notre système cognitif.

En ce quarantième anniversaire de mai 68, je ne peux qu'applaudir à l'imagination, toujours au pouvoir, chez les phonologues. Il ne faudrait pas, cependant, que cette imagination nous conduise à attribuer aux locuteurs/auditeurs d'extraordinaires talents d'architecte (comme aux termites, $c f$. Fauconnier, 1984). Nos représentations expriment des relations issues de constructions mentales faisant usage des caractéristiques physiques de la parole. Et les limites de ces relations, ce sont les contours de nos contraintes.

\section{Références bibliographiques}

Angoujard, J.P. (1997). Théorie de la syllabe. Rythme et qualité. CNRS-Editions : Paris.

Angoujard, j.P. (2005). La phonologie déclarative. In N. Nguyen, S. Wauquier-Gravelines \& J. Durand (éds) Phonologie et phonétique. Forme et substance. Hermes Science : Paris, 187-207.

Angoujard, J.P. (2006). Phonologie déclarative. CNRS-Editions : Paris.

Brandão de Carvalho, Joaquim (2004). Représentations versus contraintes. Explications formelles et substantielles en phonologie. Manuscrit non publié.

Coleman, J. (1998). Phonological Representations. Their Names, Forms and Powers. Cambridge University Press: Cambridge.

Charette, M. (1991). Conditions on Phonological Government. Cambridge University Press: Cambridge.

Fauconnier, G. (1984). Espace mentaux. Aspects de la construction du sens dans les langues naturelles.. Les Éditions de Minuit : Paris.

Grammont, M. (1894). Le patois de Franche-Montagne et en particulier de Damprichard (Franche-Comté). IV : la loi des trois consonnes. Mémoires de la Société de Linguistique de Paris, 8.

Lowenstamm, J. (1999). The beginning of word. In Phonologica 1996. Holland Academic Graphics: La Haye.

Lyche, Ch. \& Durand, J. (1996). Testing government phonology ou pourquoi le choix du schwa ? In J. Durand \& B. Laks (eds), Current Trends in Phonology. Models and Methods, ESRI, University of Salford, 443-471. 
McCarthy, J.J. (2007). Hidden Generalizations: Phonological Opacity in Optimality Theory. Equinox: London \& Oakville.

Nespor, M. \& Vogel, I. (1986). Prosodic Phonology. Foris: Dordrecht.

Nguyen, N. \& Fagyal, Z. (2003). Acoustic aspects of vowel harmony in French. In XVth International Congress of Phonetic Sciences. Barcelone, 3-9 août.

Selkirk, E. (1978). The French foot: on the statute of "mute" $e$. Studies in French Linguistics, 1-2, 79-141. 